\title{
ENTRE EL DERIVACIONISMO Y LAS DISPUTAS: LA CONFIGURACIÓN DEL SISTEMA DE Protección InTEGRAL De los DERECHOS DE NIÑOS, NIÑAS Y ADOLESCENTES EN LA CIUdAd de Villa MARÍA (CÓRDOBA) DURANTE LOS AÑOS 2014 Y 2015
}

DAIANA AILÉN MONTI ${ }^{1}$

\begin{abstract}
RESUMEN
Indagamos los particulares modos de constitución del Sistema de Protección de Derechos de niños/as y adolescentes de la ciudad de Villa María (Córdoba), durante los años 2014 y 2015. Nos interrogamos por el proceso a través del cual esta «nueva» normativa se configuró $\mathrm{y}$, además, por los modos en que convive con residuos del modelo tutelar.

Pretendemos evidenciar las tensiones y contradicciones que coexisten en los discursos y en las prácticas de los/as agentes estatales que intervienen en situaciones de vulneración de derechos de niños/as y adolescentes de clases populares.

Nuestra hipótesis reside en que, más allá de la nueva institucionalidad, aún persisten acciones ancladas en el enfoque tutelar que profundizan las condiciones de vulneración de estos/as sujetos/as.

PALABRAS CLAVE: INFANCIAS Y ADOLESCENCIAS, AGENTES ESTATALES, CLASES POPULARES.

1 Universidad Nacional de Villa María/CONICET, ciudad de Villa María, Córdoba, Argentina. Correo electrónico: daiana_monti@hotmail.com, orcid.org/0000-0002-91034964. Licenciada en Sociología de la UNVM (Universidad Nacional de Villa María). Becaria doctoral de CONICET. Estudiante del Doctorado en Ciencias Antropológicas de la UNC (Universidad Nacional de Córdoba). Docente adscripta en dos cátedras del IAPCS-UNVM: Sociología Política (Lic. en C. Política) y Antropología Social (Lic. en Sociología). Miembro de los equipos de investigación IAPCS-UNVM: «Condiciones de producción de disposiciones y experiencias infanto juveniles en barrios periféricos populares» $\mathrm{y}$ «Teorías y praxis feministas: sujetos políticos y saberes situados».
\end{abstract}




\title{
ENTRE O DERIVACIONISMO E AS DISPUTAS: A CONFIGURAÇÃO DO SISTEMA DE PROTEÇÃO INTEGRAL DOS DIREITOS DOS MENINOS, MENINAS E ADOLESCENTES NA CIDADE DE VILLA MARÍA (CÓRDOBA) DURANTE OS ANOS 2014 E 2015
}

\begin{abstract}
RESUMO
Indagamos os particulares modos de constituição do Sistema de Proteção dos Direitos dos meninos, meninas e adolescentes da cidade de Villa María (Córdoba) durante os anos 2014 e 2015. Questionamos o processo pelo qual este «novo» regulamento foi configurado e, ainda, pelas formas como convive com os resíduos do modelo tutelar. Pretendemos evidenciar as tensões e contradições que coexistem nos discursos e nas práticas dos/ das agentes estatais que intervêm em situações de vulneração dos direitos dos meninos, meninas e adolescentes das classes populares.

Nossa hipótese reside em que, para além da nova institucionalidade, ainda persistem ações ancoradas no enfoque tutelar que aprofundam as condições de vulneração deste/destas sujeitos/as.

PALAVRAS-CHAVE: INFÂNCIAS E ADOLESCÊNCIAS, AGENTES ESTATAIS, CLASSES POPULARES.

\section{BETWEEN DERIVATIONISM AND DISPUTES: THE SHAPING OF THE SYSTEM OF INTEGRAL PROTECTION OF RIGHTS OF BOYS, GIRLS AND ADOLESCENTS IN THE CITY OF VILLA MARIA (CÓRDOBA) IN 2014 AND 2015}

\begin{abstract}
We inquire into the particular ways of formation of the System of protection of rights of boys, girls and adolescents in the city of Villa María (Córdoba) in 2014 and 2015. We asked ourselves about the process from which this «new» regulation was configured, as well as for the ways of coexistence with the remains of the tutelary model.

We intend to show the tensions and contradictions coexisting in both the discourse and practice of those male and female state agents intervening in situations of infringement of rights of working-class boys, girls, and adolescents.

Our hypothesis, therefore, is that there are actions within the tutelary approach, which are ingrained beyond the new institutionality, and make the conditions of infringement of these male and female individuals deeper.
\end{abstract}

KEYWORDS: CHILDHOODS AND ADOLESCENCES, STATE AGENTS, WORKING CLASSES. 


\section{INTRODUCCIÓN: DOS PARADIGMAS EN DISCUSIÓN Y CONVIVENCIA}

La Ley Nacional 26.061, de Protección Integral de los Derechos de niños/as y adolescentes (de 0 a 18 años) se sancionó el 28 de septiembre de 2005. Fue producto de las condiciones de posibilidad que habilitó la posconvertibilidad y de luchas, disputas y negociaciones entre agentes estatales y organizaciones territoriales y gremiales de diversos lugares de nuestro país (Magistris, 2013; Morales y Magistris, 2018; Villalta, 2020). Esta ley implicó la adecuación del Estado Nacional a lo que preestablecía la Convención Internacional de los Derechos del Niño (CIDN), obligando a este a rearmarse institucionalmente para relacionarse de otro modo con las infancias y adolescencias. Este nuevo modo de relación (también objeto de luchas y disputas que llegan hasta el presente) implicó definirlas y definirlos como sujetos/as de derecho y, al mismo tiempo, redefinir el papel del Estado, de las familias y la sociedad civil en la regulación de situaciones de vulneración de sus derechos.

Este punto de vista se construyó en oposición a las maneras de intervenir sobre las infancias y las adolescencias que habilitaba la Ley Nacional 10.903, de Patronato de Menores (vigente desde octubre de 1919). Este modo de regulación de la vida de niños, niñas y adolescentes (de ahora en más NNyA) fue ampliamente estudiado por diversos autores y autoras (Cosse, 2005; Freidenraij, 2015; Stagno, 2010; Villalta, 2012; Zapiola, 2010), por ello, no nos detendremos con profundidad sobre este punto.

Sin embargo, consideramos necesario preguntarnos sobre las marcas perdurables que ha dejado este dispositivo a través del cual se construyeron, desde el Estado, dos tipos de infancias a las que les correspondieron intervenciones específicas y escindidas entre sí. Por un lado, señala Isabela Cosse (2005), a las/os NNyA con familias «normales», es decir, que ofrecían garantías para su cuidado y desarrollo, se destinaban políticas educativas (de diversas maneras, desde la escuela, se regulaba la vida de estos/as sujetos/as y de sus familias); por otro lado, para las/os «menores», como se denominaba a NNyA de sectores populares «carentes de contención» y nacidos en familias definidas como moral, económica o socialmente desvalidas o negligentes, se habilitaban 
intervenciones que permitían a diversas instituciones ${ }^{2}$ retirarles a sus padres la patria potestad y confinarlos en instituciones de beneficencia, reformatorios o entregarlos en tutela a familias «decentes». Tales medidas, enmarcadas en la Ley de Patronato, tenían como finalidad «aislar» y «corregir» a estos/as sujetos/as, en la medida en que eran concebidos como potencialmente peligrosos para la sociedad.

En relación con esto, es necesario puntualizar que tanto las infancias y las adolescencias, así como las intervenciones de las que son destinatarias, son construcciones sociales, dinámicas y variables culturalmente. Son, también, resultado de las definiciones e intervenciones por parte de profesiones y saberes expertos, como los vinculados a la medicina, a la psicología, a la pedagogía, a la psicopedagogía, a las ciencias de la educación o al trabajo social (Rustoyburu, 2016). Es decir, son categorías sociales que responden a diversos sistemas clasificatorios y a los valores dominantes sobre qué es lo apropiado o adecuado para quienes componen este grupo, así como para quienes son responsables de su cuidado y educación.

Asumiendo esta complejidad, nos preguntamos por las marcas que persisten de la Ley de Patronato en los primeros años de transición de un

${ }^{2}$ Para una información detallada sobre estas instituciones y las diversas maneras de intervenir en la vida de NNyA de sectores populares, dirigirse a: Villalta, C. (2012). «¿De quién son los niños pobres? El debate por la tutela administrativa, judicial o caritativa». En C. Villalta: Entregas y secuestros. El rol del Estado en la apropiación de niños (pp. 47-69). Buenos Aires: CELS/Ediciones del Puerto. 
paradigma a otro en la ciudad de Villa María ${ }^{3}$. En este sentido, siguiendo trabajos de otras autoras (Magistris, 2013; Villalta y Llobet, 2015; Villalta, 2020), nuestra hipótesis es que esta transición se constituyó en una instancia de convivencia entre lo que permanece del anterior modelo y lo propio de una nueva concepción que se fue instalando de manera progresiva en la comunidad y en las prácticas estatales. En este marco, pretendemos identificar cómo determinados presupuestos cognitivos e interpretativos vinculados a la ley de Patronato de Menores, todavía hoy, están presentes en la objetividad de los cuerpos y las instituciones (Bourdieu, en Villalta, 2013) que regulan las infancias y adolescencias de sectores populares.

${ }^{3}$ La ciudad de Villa María pertenece al Departamento General San Martín de la Provincia de Córdoba; se ubica en el sureste del territorio cordobés y, según datos del último censo nacional (2010), su población es de 80.000 habitantes, aproximadamente. Es una ciudad de tamaño medio y la tercera más importante de la provincia luego de Córdoba Capital y Río Cuarto. Se encuentra atravesada por la Ruta Nacional 9, que une Córdoba y Rosario, así como por la Ruta Nacional 158, que forma parte del corredor bioceánico que une parte de Brasil, Uruguay, Argentina y Chile.

Dentro del territorio villamariense, la mayor parte de sus recursos (económicos, financieros, culturales) se concentran en el centro y suroeste de la ciudad, en cambio, los barrios populares se extienden sobre el norte, noreste y este, conformando un cinturón que asume la forma de una «media luna» que rodea a los cuatro bulevares que delimitan el rectángulo céntrico del espacio urbano. Para profundizar sobre este concepto, sugerimos revisar: Truccone, D. et al. (2019). Vecinos, parientes y extraños. Lógicas de apropiación territorial en un barrio de Villa María, Córdoba. Disponible en: http://biblio.unvm.edu.ar/opac_css/doc_num.php?explnum_id $=2238$

Teniendo en cuenta estas características, el presente escrito despliega un análisis de múltiples escalas (local, provincial y nacional) que pone en diálogo las diversas acciones estatales y políticas públicas que se territorializan en esta «media luna» y sobre las vidas de los/as NNyA — y sus familias — que la habitan.

Por último, es pertinente señalar que desde el año 2018 el municipio local posee un Centro Estadístico propio que, mediante encuestas trimestrales, produce datos situados sobre la ciudad y su población. Para indagar datos actualizados sobre Villa María, revisar: Centro Estadístico, Municipalidad de Villa María, en línea: https://portalvillamaria.opendata.arcgis.com/ 
Nuestro trabajo de campo se desarrolló entre los años 2014 y 2015 en la ciudad de Villa María (Córdoba) ${ }^{4}$. Durante este tiempo realizamos entrevistas en profundidad a los/as agentes estatales municipales y provinciales que intervienen en situaciones de vulneración de derechos de NNyA de sectores populares y que, al mismo tiempo, forman parte del Sistema Local de Protección Integral de los Derechos de NNyA. Específicamente, dialogamos con funcionarios/as de la gestión del gobierno municipal (por ejemplo, de la Secretaría de Desarrollo Social, de la coordinación de las Áreas de Adolescencia, de Familia, de Educación, de la Dirección de Juventud), con integrantes del equipo local interdisciplinario de la Secretaría de Niñez, Adolescencia y Familia de la provincia de Córdoba (trabajadores/as sociales, psicólogos/as), con trabajadores/as de programas estatales municipales y provinciales que se despliegan en los territorios barriales (como trabajadores/as sociales, docentes de talleres, promotores/as de salud) y con docentes de escuelas primarias y secundarias de sectores populares de la ciudad.

En paralelo, realizamos observaciones participantes en un taller de radio dictado en una de estas instituciones escolares para acercarnos a las cotidianeidades de los/as NNyA de clases populares, con el objetivo de ponerlas en diálogo y en tensión con los puntos de vista de los/as adultos/as entrevistados. Esta estrategia - asumida como una forma de vigilancia epistemológica de nuestro propio adultocentrismo ${ }^{5}$ - nos permitió emprender un abordaje de tipo etnográfico, mediante el cual observamos los modos en que estos/as sujetos/as se relacionan e interactúan con sus pares y/o con los/as adultos/as que los/as rodean

${ }^{4}$ Esta investigación formó parte del proceso de elaboración de mi Trabajo Final de Grado para la Licenciatura en Sociología de la Universidad Nacional de Villa María, denominado: «Puntos de vista de agentes estatales municipales sobre jóvenes y adolescentes de un barrio periférico de la ciudad de Villa María (Córdoba)». A su vez, se inscribió en un proyecto de investigación financiado por la Universidad Nacional de Villa María, dirigido por la Mgter. Pavcovich, P., y titulado: «Condiciones, disposiciones y experiencias de niñas, niños, y jóvenes adolescentes de clases populares (convocatorias: 2016-2017 y 2018-2019)», cuyos objetivos generales pretendieron: a) explicar las condiciones de producción que dan cuenta de la razonabilidad de las prácticas sociales de niñas, niños y jóvenes adolescentes de clases populares y, b) comprender los puntos de vista con los que niñas, niños y jóvenes adolescentes de clases populares se relacionan con el mundo adulto.

${ }^{5}$ Para profundizar sobre el concepto de adultocentrismo, revisar: Duarte Quapper, K. (2000). ¿Juventud o Juventudes? Acerca de cómo mirar y remirar a las juventudes de nuestro continente. Última Década, 13, 59-77. https://dialnet.unirioja.es/servlet/articulo?codigo $=2256243$ 
(docentes, agentes del Estado municipal, investigadores/as). También nos permitió aproximarnos a las formas en que habitan el espacio escolar, el espacio barrial o los programas destinados a ellos/as, aunque en este escrito no abordaremos esta temática.

Los avances de este proceso de indagación, en el que evidenciamos la configuración de un entramado de múltiples tensiones y contradicciones, nos permiten comenzar a caracterizar algunas dimensiones de las sensibilidades atravesadas por la clase social, el género, la etnia y los contextos culturales (Stagno, 2011) - que se construyen desde el Estado local sobre las infancias y las adolescencias de sectores populares en una ciudad el interior de Córdoba. Al mismo tiempo, y aunque no profundicemos en esto, el enfoque que asumimos nos convoca a preguntarnos sobre cómo los/as NNyA interpelan - desde su condición etaria, de clase y de género- los dispositivos estatales de los/as que son destinatarios/as.

\section{2. ¿Cómo Pensamos el Estado?}

Este trabajo se enmarca en una perspectiva socioantropológica que nos permite distanciarnos de aquellas definiciones de Estado que lo conceptualizan como una sustancia o como un todo homogéneo (Das y Poole, 2008; Villalta, 2005). Al contrario, lo entendemos como un campo de indagación (Bourdieu, 1997, 2000), como un espacio de disputas y luchas en el que diversos/as agentes construyen múltiples modos de clasificar, categorizar $y$, en consecuencia, intervenir en la vida de NNyA en situación de vulneración de derechos, a través de dispositivos específicos como programas, proyectos o políticas públicas (Villalta, 2020).

En línea con el trabajo de Isacovich y Grinberg (2020), observamos que estas formas de intervenir se inscriben en prácticas rutinarias, consuetudinarias y en relaciones particulares que los/as agentes estatales establecen entre sí, con las familias de NNyA de sectores populares, pero también, con los usos, definiciones y redefiniciones que realizan de los documentos escritos del Estado (Das y Poole, 2008), en nuestro caso, de la Ley Nacional 26061.

Desde esta perspectiva, es dable considerar que las prácticas de estos/as agentes no nos hablan tan solo de opciones individuales. Por el contrario, forman 
parte de un sentido práctico estatal constituido a partir de un habitus ${ }^{6}$ y de una historia particular, que trae consigo una lógica propia y que no solo se objetiva en las cosas, sino que trasciende a dichos/as sujetos/as.

En este marco, recobra fuerza el desafío de pensar en aquellas marcas perdurables del paradigma tutelar — vigente por más de 80 años- que, aún hoy, forman parte de las prácticas que los/as agentes estatales despliegan cotidianamente para intervenir sobre algún aspecto de la vida de NNyA de sectores populares. A su vez, a lo largo del período estudiado, observamos que estas persistencias condicionan la adecuación al nuevo paradigma de las políticas para NNyA en la ciudad de Villa María.

Con nuestro foco puesto en la complejidad de estas relaciones, pretendemos comprender las prácticas de los/as agentes estatales en el período de transición hacia la nueva normativa, sin caer en una mirada que oponga paradigmas o señale culpables (Villalta, 2020). Más bien, nos concentramos en identificar las condiciones sociales, políticas y materiales que posibilitan estas persistencias. En este sentido, nuestro objetivo no solo se relaciona con pensar la letra de la normativa a nivel nacional, provincial y local, sino con identificar sus usos cotidianos. Retomando a Das y Poole (2008, p. 31), nos preguntamos «cómo estos documentos se encarnan» en las prácticas de los/as agentes estatales de la ciudad de Villa María y, por consiguiente, en los/as NNyA destinatarios/as de sus intervenciones.

Desde esta perspectiva, indagaremos las tensiones y disputas que suscita, entre los/as agentes de diferentes niveles estatales, la toma de decisiones e intervenciones específicas en el marco de la configuración del Sistema Local de Protección Integral de Derechos. Tensiones, vale aclarar, que son originadas en la confrontación de sus ideales sobre el cómo debe ser este dispositivo o qué responsabilidades competen a cada agente y nivel de Estado, ya sea municipal o provincial (en el próximo apartado profundizaremos sobre esto).

\footnotetext{
${ }^{6}$ En palabras de Bourdieu (2015, p. 100), el habitus como sentido práctico «es lo que permite habitar las instituciones, apropiárselas prácticamente $\mathrm{y}$, de este modo, mantenerlas activas, vivas, vigorosas, arrancarlas continuamente del estado de letra muerta, de lengua muerta, hacer revivir el sentido que se encuentra depositado en ellas, pero imponiéndoles las revisiones y transformaciones que son la contrapartida y condición de la reactivación».
} 
Asimismo, como sugerimos más arriba, a lo largo de este trabajo también nos preguntamos por las maneras en que estos/as agentes clasifican a los/as destinatarios/as de dichas intervenciones. A partir de nuestro trabajo de campo, observamos que las situaciones que se consideran dignas de intervención mediante equipos de profesionales vinculados a los saberes expertos asociados a infancias y adolescencias — son materia de intervención moral (Assusa, 2019).

Las prácticas de los/as agentes estatales están atravesadas por diversas moralidades dominantes sobre cómo son y cómo deberían ser las familias de sectores populares, sobre las crianzas o los cuidados que destinan a sus hijos e hijas, sobre sus comportamientos y sobre los parámetros para evaluar las condiciones que poseen $-\mathrm{O}$ no- para garantizar contención a los/as NNyA (Stagno, 2020; Villalta, 2012). No obstante, desde una mirada relacional, contemplamos la variedad de usos y resignificaciones que las familias de sectores populares realizan sobre estas intervenciones y dispositivos estatales (Fonseca y Cardarello, en Villalta, 2012; Leo, 2015; Stagno, 2020), sin embargo, no nos detendremos en ese análisis en la presente ocasión.

\section{LAS REGULACIONES NORMATIVAS A NIVEL PROVINCIAL Y} A NIVEL LOCAL

En este apartado pensaremos algunas dimensiones centrales de las regulaciones normativas vigentes al momento de nuestro trabajo de campo durante los años 2014 y 2015. Esto se constituye en necesario, pues, desde nuestra mirada sobre el Estado como un campo a indagar, observamos que las leyes y dispositivos legislativos que regulan la protección de derechos de NNyA no expresan ni tienen las mismas consecuencias en cada territorio. Por otra parte, es interesante reconstruir esta dimensión, en tanto, es el punto de referencia en el que se apoyan - en términos discursivos - los/as agentes entrevistados/as a lo largo de nuestra investigación. Comenzaremos por la regulación nacional para - luego de pasar por la provincial - llegar a la legislación de la ciudad de Villa María que inaugura el Sistema Local de Protección de Derechos.

Luego de la sanción de Ley Nacional 26061, las provincias que adhirieron a esta desde sus propias legislaciones, se vieron compelidas a institucionalizar, desde sus poderes Ejecutivos, los Sistemas de Promoción y Protección Integral de derechos de NNyA. Este proceso acentuó la responsabilización de la intervención local en relación con la protección y restitución de derechos. Con 
ello, cada una de las formas que asumieron estos dispositivos se asociaron a las particulares gestiones locales de los problemas de la infancia y adolescencia (Isacovich y Grinberg, 2020; Magistris, 2013).

Desde entonces, coexisten a lo largo del país una multiplicidad de modalidades de implementación de la Ley Nacional, con diversas lógicas de autonomía, diferentes políticas, programas, proyectos y actores. Al mismo tiempo, esta situación provocó una ruptura en relación con el tradicional centralismo que concentraba el Poder Judicial, anclado en la Ley de Patronato, en la regulación de las vidas de NNyA — y sus familias - en situación de vulneración de derechos (Magistris, 2013).

En relación con la provincia de Córdoba - territorio en el que se halla inscrito nuestro trabajo de campo-, la Ley provincial 9944, de Promoción y Protección Integral de los derechos de NNyA, fue sancionada en mayo de 2011 y adhirió con sus propias adecuaciones a la Ley Nacional 26061. Este dispositivo legislativo también fue resultado de un proceso paulatino y conflictivo que involucró en la discusión a diversas organizaciones de la sociedad civil y del Estado. Este punto es importante para pensar cómo se fue construyendo una particular adecuación a la Ley Nacional, no obstante, en este trabajo no nos detendremos a analizarlo.

Ahora bien, ¿qué responsabilidades delegó la provincia a las municipalidades? El artículo $\mathrm{N}^{\circ} 6$ de la ley 9944 dispuso como «autoridad de Aplicación» de la Ley y del «Sistema de Promoción y Protección Integral de los Derechos de NNyA de la Provincia de Córdoba» a la Secretaría de Niñez, Adolescencia y Familia (SENAF) hoy perteneciente al Ministerio de Justicia y Derechos Humanos de la provincia de Córdoba. Con esto se constituyó, normativamente en el ente con competencia en la elaboración e implementación de planes, programas y proyectos que promuevan el desarrollo integral de estos/as sujetos/as y sus familias.

En esta línea, el artículo $8^{\circ}$ sanciona que los organismos del Estado provincial y municipal tienen la «responsabilidad indelegable» de actuar articuladamente para garantizar el cumplimiento de las políticas públicas y el ejercicio de los derechos de NNyA. Con esto, se inauguran a lo largo y ancho de toda la provincia descentralizaciones de SENAF, denominadas Unidades de Desarrollo Regional (UDER). 
Al momento de nuestro trabajo de campo, en la provincia coexistían dieciséis UDER. La mayoría se distribuye entre las ciudades cabeceras con mayor cantidad de habitantes de los veintiséis departamentos en que se divide Córdoba. En consecuencia, observamos que algunas dependencias tienen a su cargo dos o más departamentos. Particularmente, la UDER que se encuentra en Villa María tiene bajo su responsabilidad al departamento General San Martín, que posee 127.454 habitantes (Censo Nacional INDEC, 2010), divididos en quince municipios.

Este dato nos permite señalar que la UDER local - compuesta por un delegado, un trabajador social y un psicólogo - tiene a su cargo una magnitud poblacional difícilmente abarcable a partir de tales características. Consecuentemente, a partir de nuestro trabajo de campo, observamos que la presencia de estas dependencias puede leerse en términos de una formalidad más que con un objetivo explícito (en términos de personal, recursos y presupuesto) de profundizar y sostener el Sistema de Protección Integral de NNyA.

Desde entonces, y con el condicionamiento que implican estas dificultades, la UDER se constituye normativamente en la autoridad regional encargada de actuar coordinadamente con los Servicios Locales de Promoción y Protección de Derechos (dependientes de los poderes ejecutivos municipales), a través de la adopción y aplicación de medidas de promoción, de protección integral y de excepción.

Sin el objetivo de adentrarnos extensamente en las características cada una de las medidas establecidas por la Ley 9944, vale aclarar que: a) las de promoción se vinculan a la ejecución de políticas públicas universales que garanticen el ejercicio de derechos de NNyA a nivel local; b) las de protección se activan frente a la amenaza o peligro de vulneración de los derechos de estos sujetos; y, por último, c) las de excepción son aquellas que implican la separación de NNyA de su familia, son limitadas en el tiempo y reversibles. Estas solo pueden ser determinadas por la UDER y/o SENAF y se aplican - tal como dice su nombre - de manera excepcional. Esto se debe a que el nuevo paradigma y su institucionalidad convocan, a diferencia del anterior, a que NNyA permanezcan en el seno de sus familias nucleares o extensas. Por cuestiones de espacio, en este momento no profundizaremos en las discusiones alrededor de estas medidas, sus limitaciones y/o contradicciones, así como a la centralidad que este paradigma le otorga a la idea familia. Algunos trabajos que ahondan esta línea de estudio están citados en nuestra bibliografía (Magistris, 2013; Morales y Magistris, 2018). 
Como indicamos más arriba, la ley provincial instó a cada municipio a adecuar su normativa local para hacerse cargo de promover, proteger y garantizar los derechos de NNyA. Puntualmente, se constituyeron en los responsables de garantizar los Servicios Locales de Promoción y Protección de Derechos. Con ello, deben desarrollar acciones tendientes a abarcar las medidas de promoción y protección (lo que nativamente se denomina como: niveles 1 y 2). Este trabajo debe abordarse desde la lógica del abordaje integral y a partir de la articulación con la UDER y las organizaciones de la sociedad civil vinculadas a NNyA.

La normativa que estamos revisando señala que la finalidad de articular se relaciona con potenciar los recursos existentes y establecer intervenciones integrales entre estos organismos. Nótese que a lo largo del trabajo hemos resaltado la palabra «articular»y «abordaje integral». Esto no es ingenuo, pues fueron los ejes sobre los que más contradicciones y disputas hallamos en nuestro trabajo de campo, pero sobre esto volveremos más adelante.

Para adentrarnos en el nivel local, recuperamos la Ordenanza Municipal 6363, sancionada en julio 2011. Este documento tuvo dos consecuencias a nivel local: por un lado, la adhesión del Estado municipal a la Ley Nacional 21061 y, por otro, la constitución del Sistema Local de Protección Integral de Derechos de NNyA (SPI).

Específicamente, la ordenanza señala que el SPI está conformado por: a) el área local de Protección Integral de Derechos de NNyA, que le compete al Poder Ejecutivo, a través de la Secretaría de Desarrollo Social y sus respectivas áreas (Niñez, Adolescencia y Familia) y en articulación con la UDER; b) por el Consejo Local de Infancia y Adolescencia (que hasta el momento de nuestro trabajo de campo no tuvo sistematicidad, tampoco hallamos registros oficiales que documenten las instancias en que ha funcionado, con ello, inferimos que este instrumento no ha tenido incidencia real en la problematización de cuestiones que atañen a la vida de NNyA); y, por último, c) por el Foro Local de Niños, Niñas y Adolescentes (este dispositivo tampoco tiene sistematicidad, no obstante, en nuestro trabajo de campo sí hallamos otros programas de participación de NNyA como «El Parlamento de los Niños», destinado a escuelas primarias y dependiente de la Secretaría de Educación Municipal, o el «Consejo Deliberante Estudiantil», destinado a escuelas secundarias y dependiente del Concejo Deliberante de la ciudad). 
Luego de este recorrido por las diversas normativas que han formalizado —al menos de manera parcial — el diseño de una «nueva institucionalidad» inscrita en el paradigma de la Ley 26061 en la ciudad de Villa María, es necesario aclarar que: si bien estos dispositivos significan un gran paso en el proceso de conceptualizar a NNyA como sujetos/as de derecho, rompiendo con el anterior esquema tutelar represivo, es pertinente poner nuestro foco en las transformaciones o continuidades que esta transición produjo en la práctica de los/as agentes estatales y, por tanto, en la vida de NNyA.

En esta línea, a partir de nuestro trabajo de campo observamos que los cambios en la legislación no se reflejaron automáticamente en las prácticas de los/as agentes estatales. De hecho, como mencionamos anteriormente, la transición de un paradigma a otro configuró una instancia de convivencia entre ambos. En este punto, al pensar al Estado como un espacio heterogéneo y diverso, con temporalidades y lógicas atravesadas por prácticas con una historia propia, tampoco es posible pensar que un modelo de fuerte tradición tutelar podría ser desplazado por la sola reconfiguración de medidas normativas.

Teniendo en cuenta esta complejidad y retomando diversos análisis realizados por otras autoras (Llobet, 2015; Villalta y Llobet, 2015), no desconocemos un avance innegable: la nueva Ley es producto de una disputa central en la que el Poder Judicial (mediante la intervención por parte del juez) y el Poder Ejecutivo (junto a todo su cuerpo burocrático-administrativo) se enfrentaron por definir cuestiones en torno a la protección de las infancias y adolescencias y, con ello, los términos normativos y morales en juego.

El Ejecutivo logró sustraer (no sin dificultades, negociaciones y disputas aún presentes) el monopolio de la facultad de adoptar medidas asociadas a la tutela de NNyA - fundamentalmente pobres - que concentraba el Poder Judicial (Llobet, 2015). En función de esto, delegó la tarea de protección y garantía de derechos de estos/as sujetos/as a sus organismos burocráticoadministrativos, posicionándose como «autoridad de aplicación» y procurando promover la desjudicialización de la pobreza (Villalta y Llobet, 2015). 


\section{La CONFIGURACIÓN DEL SPI EN LA CIUDAD DE VILLA MARÍA}

Poniendo en juego los razonamientos anteriores, en este apartado nos detendremos a conocer los particulares modos de constitución y desarrollo del SPI de la ciudad de Villa María, durante los años 2014 y 2015. El objetivo es realizar un análisis que identifique las contradicciones que emergen entre la nueva normativa y las prácticas concretas de los/as agentes estatales que intervienen sobre situaciones de vulneración de derechos de NNyA. Es decir, reconstruiremos los modos que esta «nueva» normativa se traduce en un espacio particular y, con ello, de qué modos convive con residuos del modelo tutelar incorporado en las instituciones vinculadas a infancias y adolescencias y, por tanto, a las personas que las habitan cotidianamente.

\subsection{LAS DIFICULTADES DE CONSTRUIR UN ABORDAJE INTEGRAL Y ARTICULADO Y EL ANHELO POR «LO ANTERIOR»: TENSIONES ENTRE LA SECRETARÍA MUNICIPAL DE DESARROLlO SOCIAL Y LA UDER}

Antes de iniciar este apartado, de acuerdo a nuestro trabajo de campo, debemos agregar otro ítem para complejizar lo que venimos diciendo: la interseccionalidad de prácticas estatales (a veces contradictorias y escindidas entre sí) sobre un mismo territorio y población, que provienen de dos niveles de Estado diferentes: el provincial (UDER) y el municipal. Tener en cuenta esto nos permite observar la variedad de intervenciones que se ponen en juego desde las diferentes instancias estatales y que, a su vez, difieren - y hasta se oponen - en sentidos y sensibilidades sobre las infancias y adolescencias. Al mismo tiempo, estas prácticas no quedan por fuera del marco general de las relaciones, tensiones y/o disputas que establecen entre sí el gobierno provincial y el municipal.

A lo largo de nuestro proceso de investigación, observamos que la mayoría de los/as agentes estatales (municipales y provinciales) que conocimos se posicionaron discursivamente desde el paradigma de protección integral. En este sentido, en cada una de las entrevistas o momentos compartidos en sus oficinas, lo primero que traían a la conversación era su perspectiva favorable a la nueva ley: iniciaban cada una de sus respuestas haciendo énfasis en esto y demostraban — repitiendo a modo de verso y de manera automática — un amplio 
conocimiento de lo dispuesto por la Ley Nacional 26061, la Ley Provincial 9944 o la Ordenanza Municipal 6363.

En este marco, si pensamos la entrevista como una relación social (Beaud y Pialoux, 2015) o como un encuentro de trayectorias sociales diversas, es posible prever que esta presentación oficial — probablemente preparada de antemano para presentarla frente a una estudiante universitaria- comenzara a resquebrajarse a medida que multiplicáramos los encuentros y profundizáramos en diversas temáticas vinculadas al SPI.

En este sentido, las primeras fricciones entre el discurso sobre la normativa y la práctica real surgieron al momento de la pregunta sobre el trabajo de articulación y el abordaje integral en la ciudad. Este es un punto de inflexión que genera constantes conflictos entre la UDER y los/as agentes de Desarrollo Social del Municipio.

A raíz de esta temática, hallamos diversos grados de disputas sobre las responsabilidades que le competen a cada ente - provincial o municipal — con respecto al SPI y las medidas de intervención definidas en la Ley 9944. De hecho, los análisis de las entrevistas y notas de campo realizadas evidencian un trabajo constante por parte de los/as agentes estatales para clasificar y definir en qué «casos» o situaciones debe intervenir cada nivel de Estado. Tal situación se profundiza a partir de las tensas relaciones (previas a las elecciones municipales, provinciales y nacionales del año 2015) que existían entre el gobierno de la provincia y el de la ciudad de Villa María. En este punto, es interesante prestar atención a cómo, en algunas ocasiones, las políticas dirigidas a NNyA quedan atrapadas en conflictos de este tipo que, en última instancia, condicionan las garantías de derechos a estos/as sujetos/as. Recuperamos la perspectiva de una agente estatal municipal:

(...) Todas las repuestas que vamos dando [en materia de promoción y protección de derechos], las vamos dando desde acá [desde el municipio] y toda esta red de protección de derechos que tenemos: SUMATE, Centros de Promoción Infantil y todos los espacios donde trabajamos te permite detectar las problemáticas de manera rápida, entonces bueno... si detectas, hay que actuar y en la actuación es donde tiene que intervenir la provincia y ahí es donde nos estamos quedando faltos de repuestas. Y esto no es por una cuestión política, por ahí digo hay algunas cuestiones que tienen que ver con la cuestión política, ¿no? Mala relación con el gobernador... pero esto no, esto es... porque no es con nosotros, sino que es con toda la provincia, con todos los municipios la falta de acciones concretas en estas cuestiones (Fragmento de entrevista realizada a 
una agente de la Secretaría Desarrollo Social de la Municipalidad de Villa María).

Esta agente estatal asume que desde el municipio se dan respuestas a la promoción y protección de derechos de NNyA, a partir de dos programas puntuales: uno denominado «SUMATE», dirigido a este grupo poblacional, cuyo objetivo es realizar actividades artísticas de recreación en los barrios populares; por otro lado, los Centros de Promoción Infantil, destinados a niños y niñas de 0 a 3 años, que garantizan guardería y talleres (de nutrición, de educación sexual, de noviazgos no violentos) para sus familias, puntualmente para sus madres. Aunque sería interesante profundizar en las modalidades de desarrollo de estos programas en los barrios populares, en esta ocasión no nos detendremos a analizarlos.

Ahora bien, la finalidad de estos programas — para esta trabajadorareside, en primer lugar, en la detección de situaciones de lo que, desde su mirada, clasifica como vulneración de derechos: «negligencia de la madre»; «estar todo el día en la calle solito», violencia familiar, abusos sexuales o malnutrición. En segundo lugar, una vez «detectado el caso», la práctica consecuente es realizar «el pedido de intervención a la UDER» para que aplique una medida de excepción.

A partir de esto, podemos identificar tres situaciones que, en la práctica de los/as agentes estatales municipales, es recurrente: primero, la acción de «detectar y derivar» sin poner en juego estrategias de intervención que medien entre un extremo y otro; segundo, un vacío en la articulación entre un nivel de Estado y otro; finalmente, la denuncia constante por parte del gobierno local de la ausencia de medidas de protección y promoción de derechos que la UDER, a través de SENAF, debería implementar.

En este punto, cabe destacar que luego de un relevamiento exhaustivo a los fines de los objetivos de mi trabajo final de grado - de la totalidad de los programas dependientes del gobierno provincial y de sus diversos ministerios, observamos que en la ciudad de Villa María no se desarrolló ninguno destinado a abordar la promoción y protección de derechos durante este período de tiempo. Tampoco hallamos una política de fortalecimiento hacia la articulación entre la UDER y el municipio para la construcción de dispositivos que puedan solventar esa ausencia. 
Una agente del Área de Adolescencia de la Secretaría de Desarrollo Social profundizó esta denuncia y señaló que — puntualmente para el rango etario con el que ella trabaja: adolescentes de sectores populares, limpiavidrios y jóvenes en conflicto con la ley penal - hay una concentración de recursos en la capital de la provincia. En este sentido, su relato retomó el punto de vista de la agente anterior, indicando que el municipio no solo se encuentra en soledad para desarrollar los dos primeros niveles de intervención, sino que, a veces realiza abordajes (como seguimientos del trayecto de escolarización, asistencia alimentaria, asistencia psicológica) que - desde su perspectivacorresponderían exclusivamente a la UDER.

¿Y cómo es esta articulación con la UDER?

La provincia no tiene programas descentralizados al interior de la provincia, ¿sí? Los programas están en Córdoba capital. Con situaciones de delito, hay un complejo que es cerrado y con otras propuestas que son proyectos socioeducativos, que son para chicos ${ }^{7}$ que están en situación de encierro, con libertad asistida y son propuestas educativas, talleres, desde lo no-formal, ¿no?... Al interior no hay nada. (...) Está el recurso humano para atención, pero no la actividad en sí.

Entonces, al no tener, por eso la UDER deriva a la $\mathrm{Muni}^{8}$. Cosa que legalmente no debería hacer porque ellos tienen que hacer seguimiento y restitución de derechos porque es un nivel superior a lo que hoy la Muni como política debería garantizar. La Muni debe garantizar políticas de prevención y promoción, y está haciendo un poco más que eso. (Fragmento de entrevista realizada a una agente del Área de Adolescencia de la Secretaría de Desarrollo Social de la Municipalidad de Villa María)

En tono con esto, otras fricciones que surgieron entre el discurso y las prácticas de los/as trabajadores/as del municipio quedaron en evidencia en relación con la prioridad que el nuevo paradigma le otorga a la estadía de NNyA con derechos vulnerados dentro de su núcleo familiar más cercano o extenso.

En múltiples y diversas entrevistas surgió la sensación de «desprotección»y «soledad» que sienten estos/as agentes cuando «detectan» situaciones que desde sus perspectivas (no desde la ley) requieren medidas

\footnotetext{
${ }^{7}$ Palabra de uso local para referirse a niños, niñas y adolescentes. En Argentina es común denominar a estos/as sujetos/as como «chicos» $\mathrm{y}$ «chicas».

${ }^{8}$ Modo abreviado, de uso cotidiano, para hacer referencia a la Municipalidad de Villa María.
} 
excepcionales y, en consecuencia, consideran necesario separar a NNyA del núcleo familiar. No obstante, como mencionamos anteriormente, a partir de lo explicitado por la Ley 9944, el Poder Ejecutivo municipal no posee la capacidad para tomar decisiones sobre las medidas de excepción: estas son territorio exclusivo de la UDER o SENAF.

En estos casos, el nuevo paradigma al que adscriben se constituye en un límite para realizar las intervenciones que — desde sus miradas - transformarían la situación de vulneración de derechos. Frente a esta imposibilidad, aparece un sentimiento de anhelo por lo anterior, por las maneras naturalizadas - en el marco de la Ley de Patronato - de resolver estas situaciones. Las palabras que pone en juego una agente estatal en el siguiente relato evidencian que «lo anterior» no solo «resolvía», sino también implicaba ciertos grados de autonomía: no había que articular, derivar o negociar con la UDER: se intervenía directamente con la jueza de menores.

En su momento, nosotros teníamos — con la Ley vieja de Patronato- un hogar, que es el Hogar Marietita ${ }^{9}$, y la provincia tenía otro, acá en Villa María, que es el Gurisito ${ }^{10}$, que ya hace varios años que no funcionan (...) Entonces, cuando había un niño vulnerado en sus derechos, la jueza venía, sacaba al chico del hogar, temporalmente lo ponía en esta institución, y bueno, nosotros éramos los tutores, hasta tanto se resolviera la situación familiar. Si era abuso o lo que fuera, se buscaba que la familia o quien se podría hacer cargo de este niño, y a partir de ahí, se restablecían los lazos familiares. (Fragmento de entrevista realizada a una agente de la Secretaría de Desarrollo Social de la Municipalidad de Villa María)

${ }^{9}$ El Hogar «La Marietita» albergaba a niños/as que estaban bajo la tutela del Poder Judicial (por diversas razones) durante la vigencia de la Ley de Patronato en el territorio cordobés. A partir de la sanción de la Ley de Protección de Derechos de Niños, Niñas y Adolescentes 9944, el gobierno provincial instó a los municipios a cerrar estos institutos. «La Marietita» no existe más desde octubre del 2014. Fuente: información extraída de un medio de comunicación local, disponible en línea: http://anteriores2.wfxgroup.com.ar/noticias/nota.asp?nid=87620

${ }^{10} \mathrm{Al}$ igual que «La Marietita», el hogar «El Gurisito» albergaba a niños/as que estaban bajo la tutela del Poder Judicial en la ciudad de Villa María. A diferencia de aquella, este hogar cerró en el año 2011. Fuente: información extraída de un medio de comunicación local, disponible en línea: https://www.eldiariodelcentrodelpais.com/2015/10/04/cerraron-institutos-pero-nodieron-otra-opcion/ 
Para reflexionar sobre esta situación, retomamos algunos análisis realizados por Carla Villalta (2012) en los que plantea que, a través de sus disputas, las diversas instituciones que intervienen en «minoridad» reclaman para sí ámbitos exclusivos de intervención (y, con ello, poder) sobre la vida de NNyA de sectores populares, que se traduce en formas de distinción, de reconocimiento social y de obtención de recursos.

A partir de esto, consideramos posible que este anhelo por el vínculo directo con la jueza de menores puede pensarse como una pérdida de poder y de exclusividad - por parte de los/as agentes estatales municipales - en las intervenciones con NNyA de sectores populares y sus familias, pero también en sus relaciones con el Juzgado de Menores y otras dependencias estatales con las que articulaban su trabajo. 
la configuración del sistema de protección integral de los derechos de niños, niñas y adolescentes en la ciudad de Villa María (Córdoba) durante los años 2014 y 2015

\section{2 ¿A QUIÉNES HAY QUE «DERIVAR» ${ }^{11}$ ?}

Si retomamos lo trabajado hasta aquí y volvemos a nuestra pregunta inicial sobre cómo funciona el SPI en Villa María, es posible señalar dos situaciones: por un lado, a la hora de intervenir en situaciones de vulneración de derechos, frecuentemente se menciona con nostalgia al accionar de «la jueza», figura central de la Ley de Patronato; por otro lado, los/as agentes municipales recurren sistemáticamente al «derivacionismo» hacia la UDER en pedido de medidas excepcionales.

En este sentido, otra pregunta que nos hicimos a lo largo de nuestro trabajo campo fue: ¿cuáles eran las situaciones que para estos/as agentes requerían una medida de excepción? Luego de analizar los diversos programas e intervenciones que llevan a cabo, observamos que se solicita intervención de la UDER frente a: a) aquellas situaciones que son caracterizadas como «riesgosas»

${ }^{11}$ Recuperamos la categoría «derivación» y/o «derivacionismo» de un artículo de Carla Villalta y Valeria Llobet (2015) referenciado en la bibliografía de este trabajo. Tal concepto les permitió a las autoras analizar algunas lógicas de las intervenciones estatales sobre NNyA en dos provincias argentinas (Mendoza y San Juan), en el marco de la adecuación de las normativas locales a la Ley Nacional 26061.

El «derivacionismo» da cuenta de una lógica particular de intervención estatal sobre las condiciones de vulneración de derechos de NNyA, cuya característica principal es la «reorientación de casos» - por parte de las áreas que reciben tales situaciones- hacia otras dependencias para que actúen, en lugar de resolverlas inmediatamente en el lugar de recepción. Esta práctica suele justificarse por diversos motivos, como, por ejemplo: la falta de recursos económicos, la escasez de personal, la explicitación de «no competencia» del espacio en cuestión o por disputas con otras dependencias a las que se les reclama la responsabilidad frente a tal situación.

El presente artículo recupera tal noción, empero, le imprime características propias de lo local que nos permiten comprender y explicar las lógicas - así como también los efectos prácticos - de las intervenciones de los/as agentes estatales villamarienses sobre las vidas de los/as NNyA que habitan la «media luna» de los barrios populares de la ciudad, en el marco de la adecuación de la normativa local hacia el paradigma de la protección de derechos.

Desde una mirada complejizadora del Estado, en nuestro caso de estudio, el derivacionsimo no puede pensarse sin su vínculo directo con el estado de las relaciones entre los gobiernos local, provincial y/o nacional, pero tampoco, sin hacer referencia a las disputas dentro del mismo municipio (en el que conviven diversos puntos de vista de los/as agentes estatales) o a los - escasos - recursos con los que cuentan las intervenciones locales de la UDER (SENAF) en relación con la concentración que prima en la capital provincial. 
para la vida de NNyA (donde hay violencia, abusos o vulneración grave de derechos), y b) por otra parte — con mayor implicancia-, «los casos» en los que se determina el fracaso de anteriores y sucesivas intervenciones del Estado municipal sobre familias de sectores populares, culpabilizando en primer término a estas por no garantizar cuidados mínimos o la «incapacidad» para «criar»a NNyA.

Este último parámetro para la intervención, que se constituye en el más frecuente, se justifica, en general, a partir de una explicación basada en la «frustración»y en la «incomprensión» de los/as entrevistados/as frente a las prácticas de las familias de sectores populares (Freidenraij, 2015): «no podemos entender» o «no comprendemos» cómo se llevan a cabo «ciertas prácticas» con respecto a NNyA.

Esta cuestión, que genera enojo e impotencia en estos/as agentes, nos permite visibilizar que las evaluaciones que realizan para clasificar e intervenir sobre NNyA en situación de pobreza y sus madres o padres descansan en «modelos» más o menos hegemónicos de familia. Al mismo tiempo, sus discursos evidencian una tendencia a moralizar hábitos y costumbres de estas, pues, desde una perspectiva sociocéntrica, no se adecúan a las obligaciones que se asocian a la «buena» maternidad y paternidad (Villalta y Llobet, 2015).

Estas marcas que perduran en los discursos y prácticas de los/as agentes construyen a las familias de sectores populares como «incapaces» e «incompetentes»y, particularmente, definen a las madres como «irresponsables» o «negligentes» (Freidenraij, 2015; Stagno, 2011). Observamos que, a través de estas construcciones, persisten, en la configuración del SPI en la ciudad de Villa María, categorías asociadas al anterior modelo tutelar y que, como sugiere Cosse (2005), los/as agentes estatales municipales aún definen a NNyA de sectores populares como sujetos/as «en peligro» o «peligrosos» y, con ello, la necesidad de «separarlos»y «aislarlos» de sus núcleos familiares para «corregir» y/o «prevenir». 


\section{3 ¿Y LA UDER? EL «DERIVACIONISMO» Y LA VIDA DE LOS/AS NNYA ENTRE INFORMES QUE VAN Y VIENEN}

Para complejizar aún más nuestro análisis, en lo que parece un juego de espejos, la delegada de la UDER, así como diversos miembros de su equipo interdisciplinario, responsabilizan al municipio de derivar continuamente «casos»allí, sin agotar las demás medidas correspondientes a los dos primeros niveles. En consecuencia, desde esta institución — erróneamente - se remarca que el SPI «solo corresponde garantizarlo al Municipio» y que, «más allá de que constantemente se requieran permisos de medidas excepcionales, si desde la UDER se decide que aún no se han agotado las medidas previas, no se actuará en consecuencia».

Bueno, el tercer nivel no es incumbencia ni del primero ni del segundo. Punto. (...) habría que desburrar ${ }^{12}$ respecto a eso. Primer y segundo nivel son competencia del municipio. SENAF es responsable del tercer nivel. Desde acá nosotros, nuestra responsabilidad es encontrar la familia, acompañar a la familia que el primer y segundo nivel nos dice. Yo supongo que, si llegan acá, han trabajado: (...) se hizo un seguimiento, hay un socioambiental y demás (...) y sepamos que el tercer nivel es nuestro, pero que tiene que ver con la consecuencia, con el abordaje que hagan en los niveles uno y dos (...)

Cuando en el área local de Niñez, Familia o como le fuera a llamar cada municipio, están agotadas esas instancias en donde el Estado debe hacerse presente, y donde la familia responsable, ahí llegamos nosotros, en el tercer nivel de abordaje, que es la medida de excepción. La medida de excepción puede resguardar del niño bajo el cuidado de una nueva familia o bajo cuidados institucionales, que esa es última instancia. (Fragmento de entrevista realizada al trabajador social del equipo interdisciplinario de la UDER-Villa María)

$* * *$

Con el abordaje que hagan, fundamentalmente, el primer y segundo nivel de los municipios, si es bueno, seguramente no se llega al tercer nivel (...) Cuando vos agotaste todas las instancias, cuando vos ya trabajaste con la familia, y no pudiste lograr nada, no podés revertir situaciones de violencia (...) maltrato o maltrato infantil o abuso, en esos casos — son casos de altísimo riesgo-, entonces, mucho no hay que trabajar primero y segundo nivel, porque hay que

12 «Desburrar» es un verbo de uso local que refiere a informar a una persona «burra», que ignora algo. 
sacarlo rápido al chico y empezar a reparar los derechos que se le han vulnerado. (Fragmento de entrevista realizada a la delegada de la UDER-Villa María)

Estos relatos refuerzan la ausencia de articulación entre estos dos niveles de Estado para garantizar los derechos de NNyA. No obstante, nos permiten hallar un punto de encuentro entre los discursos y las prácticas de los/as agentes de ambos organismos: el vacío de las medidas de protección de derechos en el marco del SPI de Villa María y, en consecuencia, el derivacionismo (Villalta y Llobet, 2015) de «casos» de un lugar a otro. Es importante resaltar que estas medidas - cuyo objetivo central es la puesta en juego de estrategias para restituir derechos ya vulnerados y evitar las medidas excepcionales - corresponden al segundo nivel de intervención e implican, normativamente, un abordaje articulado entre la UDER y el municipio.

A partir de nuestro trabajo de campo observamos que, frente a la ausencia de articulación y a las constantes disputas y desacuerdos entre los organismos de la provincia y del municipio, persisten situaciones de vulneración de derechos de NNyA de sectores populares sin ser resueltas. Es decir, hay «casos» que quedan atrapados entre las idas y vueltas de informes que el municipio realiza a la UDER solicitando medidas de excepcionales; o de la UDER rechazando estos pedidos, devolviendo los informes, explicitando que no actuará y requiriendo otras intervenciones por parte del municipio.

Esto que en el papel parece un juego de palabras, funciona como limitante del acceso a derechos de NNyA de sectores populares, a su vez — mientras estos organismos dirimen quiénes tienen la responsabilidad de intervenir-, se profundizan las condiciones de vulnerabilidad de estos/as sujetos/as. En consecuencia, identificamos que la práctica más habitual que se pone en juego desde el SPI se relaciona con determinar y definir criterios para la pertinencia o no- de las medidas excepcionales, es decir, la separación de NNyA de sus familias nucleares, lo que vuelve a evidenciar marcas perdurables del anterior sistema tutelar.

Este análisis nos permite volver a nuestra hipótesis inicial: aunque se hayan modificado — a partir de la Ley Nacional 26061 - las condiciones objetivas a nivel normativo, que estipulan una nueva institucionalidad y una nueva forma de relación entre el Estado y NNyA, hay acciones que siguen ancladas en sedimentos del anterior enfoque. A partir de esto, como sugerimos más arriba, observamos que la nueva ley no produjo mecánicamente aquello que anuncia: nuevas prácticas, nuevas costumbres y, sobre todo, nuevos grupos sociales considerados como sujetos/as de derechos. Sino, más bien, emergió un 
proceso de coexistencia entre lo nuevo y lo anterior, y con ello: disputas y negociaciones entre diversos/as agentes y organismos estatales vinculados a la protección de derechos de NNyA.

\section{A MODO DE CIERRE}

A lo largo de este trabajo indagamos los particulares modos de constitución del Sistema de Protección de Derechos de NNyA de la ciudad de Villa María (Córdoba), durante los años 2014 y 2015. Reconstruimos la forma en que esta «nueva» normativa se tradujo en este espacio particular y, con ello, de qué modos convive con residuos del anterior modelo tutelar incorporado en las instituciones vinculadas a infancias y adolescencias.

En este marco, evidenciamos las disputas, conflictos y contradicciones que coexisten en los discursos y en las prácticas (programas, proyectos, intervenciones) de los/as agentes estatales municipales y provinciales que, inscribiéndose discursivamente en el paradigma de la Ley Nacional 26061, intervienen en situaciones de vulneración de derechos de NNyA de sectores populares.

Entendiendo el Estado como un campo a indagar, recorrimos las diversas normativas que han formalizado - al menos de manera parcial— el diseño de una «nueva institucionalidad» inscrita en el paradigma de la Ley 26061 en la ciudad de Villa María, no obstante observamos que: si bien estos dispositivos significan un gran paso en el proceso de conceptualizar a NNyA como sujetos/as de derecho, rompiendo con el anterior esquema tutelar represivo, pusimos nuestro foco en las transformaciones y continuidades que esta transición produjo en la práctica cotidiana de los/as agentes estatales.

En esta línea, a partir de nuestro trabajo de campo, observamos que los cambios en la legislación no se vieron reflejados automáticamente en las prácticas de los/as agentes estatales de la ciudad de Villa María. Al contrario, la transición de un paradigma a otro configuró una instancia de convivencia entre ambos que, en algunas instancias, implica la profundizan las condiciones de vulneración de derechos de estos/as sujetos/as. 
RECIBIDO: 28 DE NOVIEMBRE DE 2020

ACEPTADO: 22 DE DICIEMBRE DE 2020

\section{BIBLIOGRAFÍA}

AssusA, G. (2019). El mito de la patria choriplanera: una sociología de la cultura del trabajo en la Argentina contemporánea. Buenos Aires: Teseopress.

BeAud, S. y PiAlouX, M. (2015). Repensar la condición obrera. Investigación en las fábricas de Peugeot de Sochaux Montbéliard. Buenos Aires: Antropofagia.

Bourdieu, P. (1997). Espíritu de Estado. Génesis y Estructura del Campo Burocrático. En Razones prácticas. Sobre la teoría de la acción (pp. 91125). Barcelona: Anagrama.

. (2000). Una interpretación de la teoría de la religión según Max Weber. En Intelectuales, política y poder (pp. 43-63). Buenos Aires: Eudeba.

. (2015). El Sentido Práctico. Buenos Aires: Siglo XXI.

Cosse, I. (2005). La infancia en los años treinta. Todo es Historia, 457(38), 4857.

DAs, V. y PoOLE, D. (2008). El estado y sus márgenes. Etnografías comparadas. Cuadernos de Antropología Social, 27, 19-52.

FREIDENRAIJ, C. (2015). «Arrastrados por el sendero del vicio». La infancia abandonada y delincuente en Buenos Aires a fines del siglo XIX y principios del XX. Boletín de la Biblioteca del Congreso de la Nación Argentina, 129, 113-124.

ISACOVICH, P. y GRINBERG, J. (Comps.). (2020). Introducción. Los derechos de las infancias y las juventudes: debates e interrogantes a la luz del 30 aniversario de la Convención de los Derechos del Niño. En Infancias y juventudes a 30 años de la Convención sobre los derechos del Niño. Políticas, normativas y prácticas en tensión (pp. 41-84). José C. Paz, Buenos Aires: EDUNPAZ, Editorial Universitaria.

LEO, M. (2015). Sobre «depósitos» y «rescates»: una aproximación cuantitativa a la circulación de niños como práctica y como estrategia familiar de los 
sectores populares. Buenos Aires, 1930-1945. 4tas Jornadas de Estudios sobre la Infancia, Buenos Aires.

Llobet, V. (2015). La infancia y su gobierno: una aproximación desde las trayectorias investigativas de Argentina. Política \& Trabalho, Revista de Ciências Sociais, 43, 37-48.

MAGISTRIS, G. (2013). La territorialización de las políticas y programas de protección de derechos de los niños, niñas y adolescentes en la provincia de Buenos Aires. X Jornadas de Sociología. Facultad de Ciencias Sociales, Universidad de Buenos Aires, Buenos Aires.

MonTI, D. (2017). Puntos de vista de agentes estatales municipales sobre jóvenes adolescentes de un barrio periférico de la ciudad de Villa María. Revista Sociales Investiga, 4, 177-192.

Morales, S. y Magistris, G. (Comps.) (2018). Niñez en movimiento. Del adultocentrismo a la emancipación. Buenos Aires: El Colectivo, Chirimbote, Ternura REVELDE.

PaVCOVICH, P. Proyecto de investigación Universidad Nacional de Villa María: «Estrategias de reproducción social e infancia(s). Experiencias de niñas, niños y adolescentes de clases populares». (Convocatorias: 2014-2015; 2016-2017).

Rustoyburu, C. (2016). Maternidad e infancia. Los consejos médicos y psicológicos en la revista ¡Hijo Mío...!, Buenos Aires, en la década de 1930. Arenal, 23(1), 117-142.

Stagno, L. (2010) Niños y jóvenes como objetos de ideas punitivas (19191943). En Una infancia aparte. La minoridad en la provincia de Buenos Aires (1930-1943) (pp. 14-54). Buenos Aires: FLACSO.

. (2011). El descubrimiento de la infancia, un proceso que aún continúa. En S. FinOcChio y N. Romero, Saberes y prácticas escolares. Rosario: Homo Sapiens Editores-FLACSO. Recuperado de: https://www.aacademica.org/leandro.stagno/4

Stagno, L. (2020). Jóvenes y familias en la intervención del Tribunal de Menores del Departamento Judicial de la Capital (La Plata, 1938-1942). Seminario virtual de discusión Familias e Infancias en Perspectiva Histórica. Grupo de Investigación Histórica Familias e Infancias en la 
Argentina Contemporánea (Instituto de Investigación de Estudios de Género, Facultad de Filosofía y Letras, Universidad de Buenos Aires).

Villalta, C. (2012). ¿De quién son los niños pobres? El debate por la tutela administrativa, judicial o caritativa. En Entregas y secuestros. El rol del Estado en la apropiación de niños (pp. 47-69). Buenos Aires: CELS/Editores del Puerto.

. (2013). Un campo de investigación: las técnicas de gestión y los dispositivos jurídico-burocráticos destinados a la infancia pobre en la Argentina. CIVITAS, 13(2) 235-258.

. (2020). Prólogo. Derivas y debates: la conformación de un campo de estudios a 30 años de la Convención sobre los Derechos del Niño. En P. ISACOVICH y J. GRINBERG, Infancias y juventudes a 30 años de la Convención sobre los derechos del Niño. Politicas, normativas y prácticas en tensión (pp. 17-26). José C. Paz, Buenos Aires: EDUNPAZ, Editorial Universitaria.

Villalta, C. y Llobet, V. (2015). Re-significando la protección. Los sistemas de protección de derechos de niños y niñas en Argentina. Revista Latinoamericana de Ciencias Sociales, Niñez y Juventud, 13(1), 167-180.

ZAPIOLA, M. C. (2010) .La Ley de Patronato de Menores de 1919. ¿Una bisagra histórica? En L. LIONETTI y D. MíGUEZ (Eds.), Las infancias en la historia argentina. Intersecciones entre prácticas, discursos e instituciones (1890-1960) (pp. 117-132). Rosario: Prohistoria.

\section{DOCUMENTOS INSTITUCIONALES}

Ley Nacional $N^{\circ}$ 26061, de Protección Integral de los Derechos de las niñas, niños y adolescentes.

Ley Provincial N 9944, de Promoción y Protección Integral de los Derechos de las niñas, niños y adolescentes en la provincia de Córdoba.

Ordenanza Municipal $N^{\circ}$ 6363, del Consejo Deliberante de la Ciudad de Villa María. 